\title{
Generalised Unitarity At One-Loop With Massive Fermions
}

\author{
S. D. Badger ${ }^{\text {** }}$

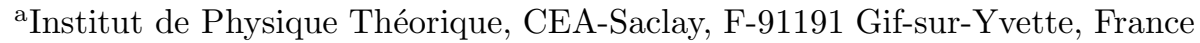 \\ E-mail: simon.badger@cea.fr
}

We describe an application of generalised unitarity to the computation of one-loop amplitudes with massive external fermions. We present analytic results for the cut-constructible parts of the leading colour contributions to the all-plus helicity configuration of the $t \bar{t} g g$ amplitude. Using a special choice for the helicity basis of the massive fermions we are able to obtain extremely compact analytic expressions. In particular we describe how one can fix the divergent contributions from tadpole and wave-function renormalisation using universal UV and IR behaviour.

\section{Introduction}

The Large Hadron Collider, due to start later this year, will require accurate $\mathrm{QCD}$ predictions for successful data analysis. New $\mathrm{TeV}$ scale physics is likely to be associated with the production and subsequent decay of heavy particles and hence background signatures will come in the form of multi-jet final states. As quantitative QCD predictions only begin at NLO the main ingredient for the computation of such backgrounds are multi-particle one-loop scattering amplitudes. Traditional Feynman methods struggle to cope with the enormous growth in the number of diagrams with the number of external legs and so many processes remain uncomputed. As an alternative, on-shell methods provide an extremely efficient tool by keeping only the physical degrees of freedom, simplifying calculations. For a review of the subject we refer the reader to reference [1].

Unitarity based techniques [2]3] have been used successfully for many years to compute complicated multi-particle and multi-loop gauge theory processes. Recent developments utilising complex momenta have lead to new recursive techniques at both tree level [4] and one-loop [5] as well as im-

\footnotetext{
*I would like to thank David Kowoser for collaboration in the early stages of this work. I am also grateful to Jurgen Körner and Zakaria Merebashvili for assistance with comparisons to their Feynman computation. Supported by Agence Nationale de Recherche grant ANR-05-BLAN0073-01.
}

proved multiple cutting techniques for logarithmic terms 6 .

A general one-loop amplitude can be written in terms of a basis of scalar box, triangle, bubble and tadpole functions together with rational terms missed by standard four-dimensional cuts. Because the quadruple cut completely freezes the loop integration, determination of the box coefficients is a completely algebraic procedure. For the lower-point integral functions Ossola, $\mathrm{Pa}$ padopoulos and Pittau have shown how to systematically generate subtraction terms to find algebraic procedures for the remaining coefficients [7. By using a special complex parametrisation of the cut momenta, Forde has demonstrated that this method can also be used to generate compact analytic expressions without subtraction [8. Initial numerical implementations, such as BlackHat 9] and Rocket [10[11, are making the first steps towards a NLO event generator.

In this paper we demonstrate the power and diversity of these new tools by computing the cutconstructable contributions to top pair production via gluon fusion. We derive compact analytic expressions in agreement with much lengthier expressions obtained from previous Feynman diagram based computations [12. This builds upon a growing body of work generalising unitarity methods to massive processes [13 14 15].

Throughout this paper all amplitudes are considered to be colour-ordered helicity amplitudes, 


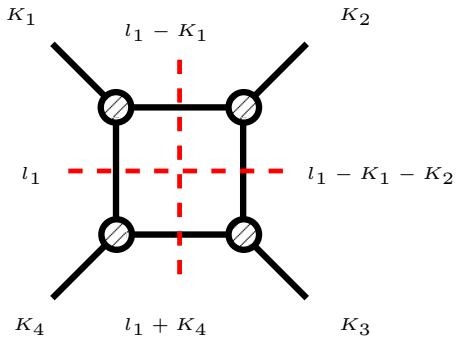

Figure 1. Quadruple cut for box coefficients with all momenta out-going and loop momentum flowing clockwise.

using the standard spinor-helicity formalism to write the amplitudes in terms of spinor products. We accommodate massive momenta into the formalism by decomposing them into two massless momenta using an arbitrary massless reference vector, $\eta[16]$ :

$p=p^{b}+\frac{m_{p}^{2}}{\langle\eta|p| \eta]} \eta$.

This then allows the definition of external fermion wavefunctions as follows [17,18,

$u_{ \pm}(q, m)=\frac{(q+m)|\eta \mp\rangle}{\left\langle q^{\mathrm{b}} \pm \mid \eta \mp\right\rangle}$,

$v_{ \pm}(q, m)=\frac{(q-m)|\eta \mp\rangle}{\left\langle q^{\mathrm{b}} \pm \mid \eta \mp\right\rangle}$.

\section{Integral Coefficients with massive prop- agators}

In this section we outline the method for direct extraction of integral coefficients with arbitrary internal and external masses 8113. Throughout the next section we take all external momenta to be out-going and all loop momenta to circulate in a clockwise direction.

\subsection{Box Coefficients}

For the coefficients of the box integrals, the onshell constraints on the loop momenta freeze the loop integration resulting in a purely algebraic procedure for the evaluation of the quadruple cut 6. Here we use a loop momentum parametrised by

$l_{1}=a K_{4}{ }^{\mathrm{b}}+b K_{1}{ }^{\mathrm{b}}+c\left|K_{4}{ }^{\mathrm{b}}\right\rangle\left[K_{1}{ }^{\mathrm{b}}|+d| K_{1}{ }^{\mathrm{b}}\right\rangle\left[K_{4}^{\mathrm{b}} \mid\right.$,

where

$K_{4}{ }^{\mathrm{b}}=\frac{\gamma K_{4}-S_{4} K_{1}}{\gamma^{2}-S_{1} S_{4}}$,
$K_{1}{ }^{\mathrm{b}}=\frac{\gamma K_{1}-S_{1} K_{4}}{\gamma^{2}-S_{1} S_{4}}$,
$\gamma=K_{1} \cdot K_{4} \pm \sqrt{\left(K_{1} \cdot K_{4}\right)^{2}-S_{1} S_{4}}$.

One can find the values of $a, b, c, d$ from the constraints,

$$
\begin{aligned}
\mathcal{S}=\{ & l_{1}^{2}=m_{1}^{2},\left(l_{1}-K_{1}\right)^{2}=m_{2}^{2}, \\
& \left.\left(l_{1}-K_{1}-K_{2}\right)^{2}=m_{3}^{2},\left(l_{1}+K_{4}\right)^{2}=m_{4}^{2}\right\} ;
\end{aligned}
$$

explicitly this gives us

$$
\begin{array}{ll}
a & =\frac{S_{1} \widehat{S}_{4}+\gamma \widehat{S}_{1}}{\gamma^{2}-S_{1} S_{4}}, \\
d & =\frac{1}{c}\left(a b-\frac{\mu^{2}}{\gamma}\right),
\end{array}
$$

and $c$ as a solution to the quadratic equation:

$$
\begin{aligned}
& c^{2}\left\langle K_{4}{ }^{b}\left|K_{2}\right| K_{1}{ }^{b}\right]+\left(a b-\frac{m_{1}^{2}}{\gamma}\right)\left\langle K_{1}{ }^{b}\left|K_{2}\right| K_{4}{ }^{b}\right] \\
& +c\left(a\left\langle K_{4}{ }^{b}\left|K_{2}\right| K_{4}{ }^{b}\right]+b\left\langle K_{1}^{b}\left|K_{2}\right| K_{1}^{b}\right]-\widehat{S}_{12}\right)=0 .
\end{aligned}
$$

Here the mass dependence is determined by $\widehat{S}_{4}=$ $S_{4}+m_{1}^{2}-m_{4}^{2}, \widehat{S}_{1}=S_{1}+m_{1}^{2}-m_{2}^{2}$ and $\widehat{S}_{12}=$ $S_{2}+2 K_{1} \cdot K_{2}+m_{2}^{2}-m_{3}^{2}$. We label the complex solutions for the loop momentum as $l_{1}^{ \pm}$, corresponding to the solutions $c_{ \pm}$of eq. (11). The value of the box coefficient is then given simply as the product of four tree amplitudes evaluated at the values, $l_{1}^{ \pm}$, of the loop momentum:

$C_{4 ; K_{1}\left|K_{2}\right| K_{3}}^{m_{1} m_{1} m_{2} m_{3} m_{4}}=\frac{i}{2} \sum_{\sigma= \pm} A_{1} A_{2} A_{3} A_{4}\left(l_{1}^{\sigma}\right)$. 


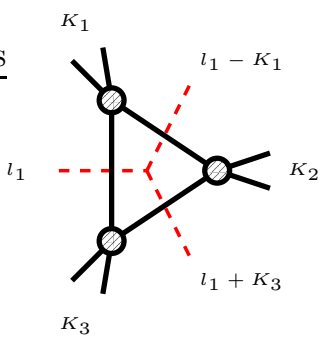

Figure 2. Generic triangle cut diagram

\subsection{Triangle Coefficients}

For the triple cuts we are left with a non-trivial one-dimensional integral after the on-shell conditions have been imposed. Following the formalism of Forde 8 , we can use simple complex analysis to isolate the scalar triangle coefficients, and again reduce the problem to a purely algebraic one. We first write down a generic triple cut integral as

$$
T_{3}=\frac{8 i \pi^{3}}{\pi^{D / 2}} \int d^{D} l_{1} \prod_{i=1}^{3} \delta\left(l_{i}^{2}-m_{i}^{2}\right) A_{1} A_{2} A_{3} .
$$

The loop momentum is chosen in a similar way to the box case but now has a free a complex parameter, $t$ :

$l_{1}=a K_{3}{ }^{b}+b K_{1}{ }^{b}+t\left|K_{3}{ }^{b}\right\rangle\left[K_{1}{ }^{b}\left|+\frac{c}{t}\right| K_{1}{ }^{b}\right\rangle\left[K_{3}{ }^{b} \mid\right.$,

where $K_{1}^{b}, K_{3}{ }^{b}$ are defined analogously to eqs. (5-7). The triple cut integral can then be written in terms of its pole structure in the complex $t$ plane,

$$
\begin{array}{r}
T_{3}=\frac{4 i \pi^{3}}{\pi^{D / 2}} \int J_{t} d t \sum_{\sigma}\left(\operatorname{Inf}_{t}\left[A_{1} A_{2} A_{3}\left(l_{1}^{\sigma}\right)\right]\right. \\
\left.+\frac{\operatorname{Res}_{t=t_{i}}\left(A_{1} A_{2} A_{3}\left(l_{1}^{\sigma}\right)\right.}{t-t_{i}}\right)
\end{array}
$$

where the $\operatorname{Inf}_{t}$ operation encodes the polynomial behaviour on the boundary of the $t$ contour. The loop momenta, $l^{\sigma}$, are the complex solutions to the on-shell conditions,

$$
\mathcal{S}=\left\{l_{1}^{2}=m_{1}^{2},\left(l_{1}-K_{1}\right)^{2}=m_{2}^{2},\left(l_{1}+K_{3}\right)^{2}=m_{3}^{2}\right\} .
$$

Because the second term in the above equation has no $t$ dependence in the numerator and has at least one additional propagator it must be associated with the scalar box coefficients which have already been determined. Therefore the only contribution to the scalar triangle is the boundary behaviour described by the large $t$ polynomial, $\operatorname{Inf}_{t}$. For the given choice of parametrisation, the integrals over positive and negative powers of $t$ vanish:

$\int d t J_{t} t^{n}=0 \quad \forall n \in \mathbb{Z} /\{0\}$.

We are then only left with the $t^{0}$ component which forms the complete coefficient,

$C_{4 ; K_{1} \mid K_{2}}^{m_{1} m_{2} m_{3}}=-\left.\frac{1}{2} \sum_{\sigma} \operatorname{Inf}_{t}\left[A_{1} A_{2} A_{3}\left(l_{1}^{\sigma}\right)\right]\right|_{t^{0}}$.

\subsection{Bubble Coefficients}

The bubble coefficients can be extracted from the double cut using a similar analysis to the triple cut case, as considered in the previous section. In this case two non-trivial integrals remain, which we choose to be parametrised by $y$ and $t$. The basis for the loop momentum in this case requires the introduction of an arbitrary massless
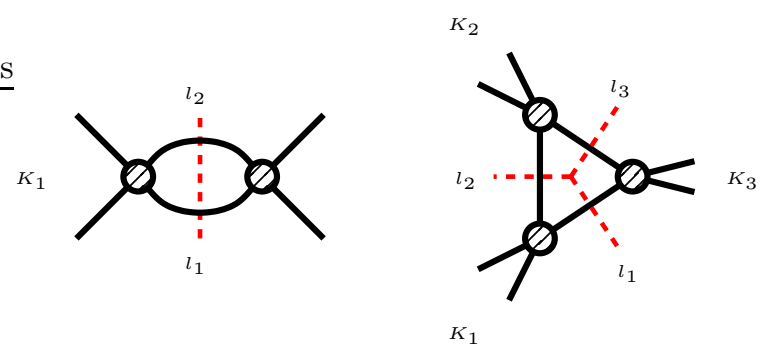

Figure 3. Bubble cuts with triangle subtraction terms 
vector $\chi$ for which the final coefficient is independent,

$$
\begin{aligned}
& l_{1}=y K_{1}{ }^{b}+a(1-y) \chi+t\left|K_{1}{ }^{b}\right\rangle[\chi \mid \\
& +\frac{b y(1-y)}{t}|\chi\rangle\left[K_{1}{ }^{b} \mid,\right.
\end{aligned}
$$

where

$K_{1}^{b}=K_{1}-\frac{S_{1}}{\bar{\gamma}} \chi, \quad \bar{\gamma}=\left\langle\chi\left|K_{1}\right| \chi\right]$.

The coefficients $a$ and $b$ are solutions to the onshell constraints,

$$
\left\{l_{1}^{2}=m_{1}^{2},\left(l_{1}-K_{1}\right)^{2}=m_{2}^{2}\right\} .
$$

We find that there are non-vanishing integrals over both $y$ and $t$ which complicates the procedure and requires both pure bubble and triangle subtraction terms to be included. The final formula for the coefficient is relatively simple however,

$$
\begin{aligned}
& C_{2 ; K_{1}}^{m_{1} m_{2}}=-\left.i \operatorname{Inf}_{t}\left[\operatorname{Inf}_{y}\left[A_{1} A_{2}\left(l_{1}^{\sigma}\right)\right]\right]\right|_{y^{i} \rightarrow Y_{i}, t^{0}} \\
& -\frac{1}{2} \sum_{\left\{K_{2}\right\}} \sum_{y=y_{ \pm}} \operatorname{Inf}_{t}\left[\left.\operatorname{Res}_{y}\left[A_{1} A_{2} A_{3}\left(l_{1}^{\sigma}, y_{ \pm}\right)\right]\right|_{t^{i} \rightarrow T_{i}},\right.
\end{aligned}
$$

where $y_{ \pm}$are the two solutions to the additional triple cut constraint $\left\{\left(l_{1}+K_{3}\right)^{2}=m_{3}^{2}\right\}$. The explicit values for these non-vanishing integrals can be found in references [81319, although some care must be taken when dealing with the different momentum conservation conventions.

\section{Application to top pair production}

The four-point amplitudes for top pair production through gluon fusion has were computed long ago using Feynman techniques [20] and presented analytically more recently [12]. As a test of our method for extracting integral coefficients for arbitrary internal and external masses we recompute the cut-constructable parts of the colour ordered primitive amplitudes, $A_{4}^{[L]}$ and $A_{4}^{[R]}$, defined by:

$A_{4}^{(1)}\left(1_{Q}, 2,3,4_{Q}\right)=A_{4}^{[L]}\left(1_{Q}, 2,3,4_{Q}\right)$

$+\frac{1}{N_{c}^{2}} A_{4}^{[R]}\left(1_{Q}, 2,3,4_{Q}\right)+$ fermion/scalar loops

+ rational terms.
The left-moving primitive amplitudes are those in which the fermion turns left around the loop and the right-moving amplitudes where it turns right. The full integral basis is shown in figure 4. In terms of the integral basis, the cut-constructible parts of the primitive amplitudes are:

$$
\begin{aligned}
& A_{4}^{[L]}\left(1_{Q}, 2,3,4_{Q}\right)=C_{4}^{000 m} I_{4}^{000 m} \\
& +C_{3 ; 12}^{00 m} I_{3}^{00 m}+C_{3 ; 23}^{00 m} I_{3}^{00 m}+C_{3 ; 23}^{0 m 0} I_{3}^{0 m 0} \\
& +C_{3 ; 23}^{000} I_{3}^{000}+C_{2 ; 12}^{0 m} I_{2}^{0 m}+C_{2 ; 23}^{00} I_{2}^{00} \\
& +\frac{c_{\Gamma} C_{1}}{\epsilon}\left(\frac{\mu^{2}}{m^{2}}\right)^{\epsilon} \\
& A_{4}^{[R]}\left(1_{Q}, 2,3,4_{Q}\right)=C_{4}^{m m m 0} I_{4}^{m m m 0} \\
& +C_{3 ; 12}^{m m 0} I_{3}^{m m 0}+C_{3 ; 23}^{m m 0} I_{3}^{m m 0}+C_{3 ; 23}^{m 0 m} I_{3}^{m 0 m} \\
& +C_{3 ; 23}^{m m m} I_{3}^{m m m}+C_{2 ; 12}^{m 0} I_{2}^{0 m}+C_{2 ; 23}^{m m} I_{2}^{m m} \\
& +\frac{c_{\Gamma} C_{1}^{\prime}}{\epsilon}\left(\frac{\mu^{2}}{m^{2}}\right)^{\epsilon}
\end{aligned}
$$

The compact tree level input for each coefficient can be generated through on-shell recursion relations [4. The procedure for computing the coefficient is easily automated and has been done both numerically and analytically. The analytic form of the final coefficient strongly depends on the various choices for the reference momenta of both internal and external particles. In order find the most compact representations it is convenient to begin with the most general representation and use complex reference momenta.

Here we present the full results for the all-plus helicity configuration, the remaining helicity amplitudes will be presented elsewhere. In this simple case the coefficients $C_{3 ; 23}^{000}, C_{3 ; 23}^{m m m}, C_{3 ; 23}^{0 m 0}, C_{3 ; 12}^{00 m}, C_{3 ; 34}^{00 m}, C_{2 ; 23}^{00}$ and $C_{2 ; 23}^{m m}$, all vanish leaving only 7 out of the 14 non-zero. In fact we can further simplify the solution by making a special choice for the reference momenta $\eta_{1}, \eta_{4}$. Choosing $\eta_{1}=\eta_{4}=p_{2}$ also makes the $C_{4}^{000 m}$ coefficients vanish and the 6 non zero coefficients are:

$$
\begin{aligned}
& C_{4}^{m m m 0}\left(1_{Q}^{+}, 2^{+}, 3^{+}, 4_{Q}^{+}\right)= \\
& -\frac{i m^{3}\langle 2|1| 2][32]\left(2\langle 2|1| 2]+s_{23}\right)}{2\left\langle 21^{b}\right\rangle\left\langle 24^{b}\right\rangle\langle 3|1| 2]}
\end{aligned}
$$




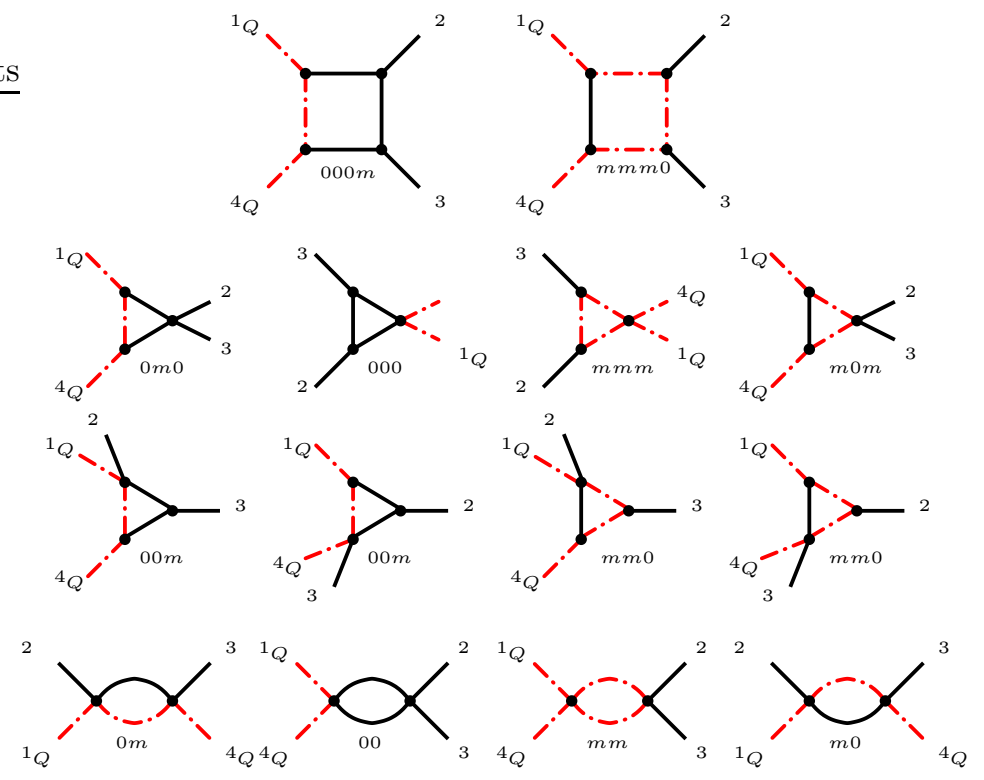

Figure 4. The scalar integral basis for the $t \bar{t} g g$ amplitude. The red dot-dashed lines represent massive fermions whereas the solid black lines represent gluons. The internal mass labels appear as superscripts, i.e. $C_{4}^{000 m}$

$$
\begin{gathered}
C_{3 ; 23}^{m 0 m}\left(1_{Q}^{+}, 2^{+}, 3^{+}, 4_{Q}^{+}\right)= \\
\frac{i m^{3}\left(s_{23}+2\langle 2|1| 2]\right)[32]}{2\left\langle 21^{b}\right\rangle\left\langle 24^{b}\right\rangle\langle 3|1| 2]} \\
C_{3 ; 12}^{m m 0}\left(1_{Q}^{+}, 2^{+}, 3^{+}, 4_{Q}^{+}\right)= \\
\quad-\frac{i m^{3}\left(\langle 2|1| 2]^{2}-2\langle 2|1| 3]\langle 3|1| 2]\right)[32]}{2\left\langle 21^{b}\right\rangle\left\langle 24^{b}\right\rangle\langle 2|1| 2]\langle 3|1| 2]} \\
C_{3 ; 34}^{m m 0}\left(1_{Q}^{+}, 2^{+}, 3^{+}, 4_{Q}^{+}\right)= \\
-\frac{i m^{3}\langle 2|1| 2][32]}{2\left\langle 21^{b}\right\rangle\left\langle 24^{b}\right\rangle\langle 3|1| 2]} \\
C_{2 ; 12}^{0 m}\left(1_{Q}^{+}, 2^{+}, 3^{+}, 4_{Q}^{+}\right)= \\
\frac{i m^{3}\langle 2|1| 3][23]}{\left\langle 21^{b}\right\rangle\left\langle 24^{b}\right\rangle\langle 2|1| 2]^{2}} \\
C_{2 ; 12}^{m 0}\left(1_{Q}^{+}, 2^{+}, 3^{+}, 4_{Q}^{+}\right)= \\
-C_{2 ; 12}^{0 m}\left(1_{Q}^{+}, 2^{+}, 3^{+}, 4_{Q}^{+}\right) .
\end{gathered}
$$

All of these expressions agree numerically with expressions extracted from the Feynman calculation of ref. [12].

\subsection{Fixing the tadpole and wave-function renormalisation coefficients}

The final step in the calculation is to fix the coefficients of the remaining $\frac{1}{\epsilon}$ terms coming from tadpole and wavefunction renormalisation contributions. Although it may be possible to evaluate these terms using unitarity cuts, a number of subtleties arise, as has been discussed in a recent numerical study 21. Instead we find it more practical to use the universal UV and IR factorisation properties [22 23].

Here we present a simple argument which is sufficient to find the remaining cut-constructible terms for the $t \bar{t} g g$ amplitude. A complete analysis will appear elsewhere [19]. The first step is to notice that the pure $\frac{1}{\epsilon}$ poles must be proportional to the tree level amplitude, $A_{4}^{(0)}$. Since we know the analytic forms for the bubble integrals, and their coefficients this allows us to write down the coefficients of the remaining $\log \left(m^{2}\right)$ terms. From the basis for the primitive amplitudes, eqs. 24. 
25), we see these $\log \left(m^{2}\right)$ terms can be completely determined from the coefficient of the pure $\frac{1}{\epsilon}$ pole,

$C_{1}=\alpha A_{4}^{(0)}-C_{2 ; 12}^{0 m}-C_{2 ; 23}^{00}$

$C_{1}^{\prime}=\alpha^{\prime} A_{4}^{(0)}-C_{2 ; 12}^{m 0}-C_{2 ; 23}^{m m}$.

The coefficients $\alpha$ and $\alpha^{\prime}$ are simply derived from the well known UV behaviour of the massless amplitudes and the recent small mass factorisation of Moch and Mitov 23. The factorisation of the massive loop amplitude, $A^{(1)}(m)$, is given by,

$A^{(1)}(m) \stackrel{m \rightarrow 0}{\rightarrow} A^{(1)}(0)+\sum_{i=1}^{n} Z_{2}^{[f(i)]} A^{(0)}(0)$

From the known pole structure of the massless amplitude, $A^{(1)}(0)$, we find a contribution of $-\frac{3 n_{Q} C_{F}}{2}[2425]$ to the $1 / \epsilon$ coefficient. It is then straightforward to read off an additional factor of $-C_{F} n_{Q}$ from $Z_{2}^{[Q]}$ for each heavy quark appearing in the amplitude 2 Putting the two pieces of information together gives us:

$\alpha=-\alpha^{\prime}=-\frac{5}{2}$,

which matches the known result [12].

\section{Conclusions}

We have shown that using the unitarity formalism of Forde [8, and its generalisation for arbitrary masses [13, provides an efficient method for calculations of complicated QCD processes. We presented a simple example of a helicity amplitude for the process of top pair production through gluon fusion while we refer the reader to [19] for a complete analysis.

We foresee that future developments to include rational contributions [10|26]9] should permit applications to much needed processes of top production in association with many jets in the near future 21.

${ }^{2}$ There will also be gluon self energy corrections, $Z_{2}^{[g]}$, but they appear in heavy quark primitive amplitudes not considered here.

\section{REFERENCES}

1. Z. Bern, L.J. Dixon and D.A. Kosower, Annals Phys. 322 (2007) 1587, 0704.2798.

2. Z. Bern et al., Nucl. Phys. B425 (1994) 217, hep-ph/9403226.

3. Z. Bern et al., Nucl. Phys. B435 (1995) 59, hep-ph/9409265.

4. R. Britto, F. Cachazo and B. Feng, Nucl. Phys. B715 (2005) 499, hep-th/0412308.

5. Z. Bern, L.J. Dixon and D.A. Kosower, Phys. Rev. D71 (2005) 105013, hep-th/0501240.

6. R. Britto, F. Cachazo and B. Feng, Nucl. Phys. B725 (2005) 275, hep-th/0412103.

7. G. Ossola et al., Nucl. Phys. B763 (2007) 147, hep-ph/0609007.

8. D. Forde, Phys. Rev. D75 (2007), 0704.1835.

9. C.F. Berger et al., (2008), 0803.4180.

10. W.T. Giele, Z. Kunszt and K. Melnikov, JHEP 04 (2008) 049, 0801.2237.

11. W.T. Giele and G. Zanderighi, (2008), 0805.2152.

12. J.G. Korner and Z. Merebashvili, Phys. Rev. D66 (2002) 054023, hep-ph/0207054.

13. W.B. Kilgore, (2007), 0711.5015.

14. R. Britto and B. Feng, Phys. Rev. D75 (2007) 105006, hep-ph/0612089.

15. R. Britto, B. Feng and P. Mastrolia, (2008), 0803.1989.

16. R. Kleiss and W.J. Stirling, Nucl. Phys. B262 (1985) 235.

17. C. Schwinn and S. Weinzierl, JHEP 04 (2007) 072, hep-ph/0703021.

18. G. Rodrigo, JHEP 09 (2005) 079, hep$\mathrm{ph} / 0508138$.

19. S.D. Badger, in preparation .

20. W. Beenakker et al., Phys. Rev. D40 (1989).

21. R.K. Ellis et al., (2008), 0806.3467.

22. Z. Bern and A.G. Morgan, Nucl. Phys. B467 (1996) 479, hep-ph/9511336.

23. A. Mitov and S. Moch, JHEP 05 (2007) 001, hep-ph/0612149.

24. W.T. Giele and E.W.N. Glover, Phys. Rev. D46 (1992) 1980.

25. S. Catani and M.H. Seymour, Nucl. Phys. B485 (1997) 291, hep-ph/9605323.

26. G. Ossola et al., JHEP 05 (2008) 004, 0802.1876 . 